\title{
Essais
}

ESSAIS

Revue interdisciplinaire d'Humanités

Hors-série 6 | 2021

Agrobiodiversité et territoires

\section{La poule Coucou de Rennes : de la conservation du patrimoine à sa valorisation économique}

Rencontre avec Jean-Luc Maillard

The Rennes cuckoo chicken: from heritage conservation to economic development

Meeting with Jean-Luc Maillard

\section{OpenEdition}

Journals

Édition électronique

URL : https://journals.openedition.org/essais/7956

DOI : $10.4000 /$ essais. 7956

ISSN : 2276-0970

Éditeur

École doctorale Montaigne Humanités

Édition imprimée

Date de publication : 1 mars 2021

Pagination : 181-186

ISBN : 978-2-492780-00-4

ISSN : $2417-4211$

Référence électronique

"La poule Coucou de Rennes : de la conservation du patrimoine à sa valorisation économique ", Essais [En ligne], Hors-série 6 | 2021, mis en ligne le 16 mars 2021, consulté le 18 janvier 2023. URL: http://journals.openedition.org/essais/7956 ; DOI : https://doi.org/10.4000/essais.7956 


\title{
La poule Coucou de Rennes : de la conservation du patrimoine à sa valorisation économique
}

\author{
Entretien avec Jean-Luc Maillard ${ }^{1}$
}

Jean-Luc Maillard est directeur et conservateur en chef de l'écomusée du pays de Rennes. Cet écomusée est un établissement culturel de Rennes Métropole qui a la particularité de disposer, outre ses expositions permanentes et temporaires, d'un site d'une vingtaine d'hectares qui présente l'agriculture bretonne, d'un cheptel conservatoire d'une vingtaine de races et de vergers patrimoniaux. Il pratique donc une conservation de la biodiversité domestique qui motive cette intervention.

Le propos vise à relater une action de conservation-valorisation qui s'inscrit dans la durée. En effet, celle-ci s'étire sur près de 20 ans et je suis aujourd'hui convaincu que s'il n'y avait pas eu ce "temps donné au temps", nous n'aurions pas ancré cette opération en profondeur et assuré sa réussite.

Il n'y a pas eu, en tout cas au départ, "l'intelligence » de l'opération, c'est à dire une vision globale et organisée qui nous aurait donné un plan d'action. Nous avons fonctionné par l'intuition, par l'écoute et le bon sens, le hasard a fait le reste avec des partenaires compétents, motivés et engagés. Aujourd'hui, il est souvent dit que la Coucou de Rennes est un des rares exemples de valorisation "réussie " d'une ancienne race locale de volaille, en conciliant conservation et production sans compromettre le patrimoine génétique de la race. Ce constat satisfaisant amène à comprendre les causes de cette réussite, à décrypter les différentes phases de l'opération et définir ainsi les possibilités de reproductibilité et les facteurs limitants.

La Coucou de Rennes est une race de volaille dont l'histoire ne date pas d'hier. Avec beaucoup d'humilité, notre action se situe dans la longue lignée de la sélection animale et végétale française. En effet, le milieu du XIX ${ }^{\mathrm{e}}$ siècle voit l'avènement des races et variétés domestiques. Qu'il s'agisse de volailles, de bovins ou de fruits et légumes, la sélection est une préoccupation où les sociétés savantes, les sociétés d'agriculture et d'horticulture rassemblent des dignitaires, des chercheurs, tous passionnés par les sciences naturelles et «l'acclimatation ». $\grave{A}$ 
Rennes, dans les années 1880, un certain docteur Ramé s'intéresse aux volailles, en particulier à une population de poules dite "Coucou de Rennes ", qu'il remarque dans les poulaillers de Haute-Bretagne. Ce notable rennais, aviculteur éclairé, très investi au sein de la Société d'Aviculture de France, devient assez influent pour pouvoir inscrire la Coucou de Rennes au registre des grandes races françaises. Après plus de 30 ans, ses efforts seront couronnés de succès puisque la race et son «standard » seront officiellement reconnus en 1914.

Malgré ses qualités et sa popularité, les années 1950-1960 sonnent le glas pour la Coucou comme pour toutes les grandes races de volailles françaises. Avec l'agriculture productiviste, de nouvelles races ou de nouvelles variétés apparaissent, beaucoup plus productives, et en général très spécialisées. Alors que la Coucou de Rennes, comme les autres races fermières traditionnelles, est une volaille " mixte " (qui produit des œufs et une bonne chair), de nouvelles « lignées » spécialisées envahissent les élevages français et " balaient » la bassecour traditionnelle nationale... Conséquence inéluctable, au début des années 1980, la disparition de la Coucou de Rennes est annoncée et ses éleveurs ont disparu. Ainsi commence notre action, celle d'un sauvetage patrimonial doublé d'une valorisation économique.

Nous étions un peu sceptiques à l'Ecomusée sur la disparition définitive de la volaille. Commence alors une longue recherche, ponctuée de nombreuses impasses, jusqu'à la découverte d'un dernier éleveur qui nous confia une partie de son cheptel-relique. Avec cette race retrouvée, nous eûmes la sensation de détenir un trésor fragile dont nous devions assurer la pérennité. Nous ne pensions pas alors à sa valorisation économique mais plutôt à sa conservation. Détenteurs d'un tel patrimoine, nous devions faire en sorte qu'il soit disponible pour les générations futures, démarche professionnelle fréquente dans les écomusées et les parcs naturels régionaux. Nous nous sommes donc empressés de créer une association d'éleveurs amateurs, groupement de personnes motivées parce qu'ils étaient aviculteurs de loisirs ou intéressés par le patrimoine et la biodiversité domestique. Cette association d'éleveurs amateurs eut pour finalité de multiplier les reproducteurs et surtout de multiplier les élevages pour faire en sorte qu'un problème sanitaire ne puisse faire disparaitre ce cheptel "relique ".

Après dix ans de multiplication de l'effectif de la race, une nouvelle association se crée, dédiée à la production de volailles de consommation, c'està-dire un groupement d'éleveurs dotés d'un projet professionnel. Ce sont ces agriculteurs, en recherche de diversification agricole et de production de qualité, qui vont donner la dimension réellement économique du projet en créant une micro-filière d'une quinzaine d'éleveurs et permettre le retour de la Coucou de Rennes sur les marchés rennais et alentours.

Très vite, un constat s'impose : la Coucou de Rennes dispose d'un véritable capital de sympathie et une mémoire vive de cette volaille subsiste. Précisons ici que Rennes est une ville dotée d'une sociologie rurale forte, avec une ancienne 
bourgeoisie urbaine vivant de la terre et de ses revenus. Il y a ici une mémoire rurale et agricole, un terreau favorable à notre initiative. Cet attachement à la ruralité se concrétise encore aujourd'hui par la subsistance d'une grande tradition rennaise : le marché des Lices. Ce grand marché alimentaire de producteurs de proximité constitue une véritable institution métropolitaine, un lieu d'achat autant qu'un motif de sortie. C'est là que se croisent les grandes toques gastronomiques d'Ille-et-Vilaine et ces derniers auront une influence considérable dans notre aventure... En termes d'organisation du projet, le schéma initial est parlant : un écomusée avec des éleveurs amateurs qui ont comme préoccupation initiale la conservation. Avec sa notoriété, l'Écomusée s'avère vite être la caution historique et scientifique de cette aventure, lui-même étant le détenteur du patrimoine génétique initial et incontestable.

Autre phase intéressante de l'opération, celle du passage d'une association de protecteurs amateurs à une association de producteurs professionnels ayant pour objectif de créer une micro-filière économique. Cela ne coulait pas de source et nous étions tous assez dépourvus devant ce nouveau cap à franchir. Les constats sont alors évidents : notre patrimoine génétique est précieux et tout indique qu'il s'agit aussi d'un capital gastronomique exceptionnel mais comment faire ? L'élément déterminant arrive presque par hasard, en 1992, avec une initiative nationale originale : l'Inventaire du patrimoine culinaire de la France réalisé par le Centre National des Arts Culinaires (aujourd'hui disparu). Cet Inventaire doit dresser une liste exhaustive des produits spécifiques par région. Chaque région aura donc un tome dont les contenus sont supervisés par des scientifiques indépendants et suffisamment éloignés des intérêts locaux. Philippe Marchenay, que nous avions sollicité un an auparavant dans le cadre de nos recherches, reprend alors contact avec nous en s'étonnant de ne pas voir notre volaille bretonne figurer dans la première liste des produits recensés... Ce chercheur, ethnobiologiste et éco-anthropologue, animait avec Laurence Bérard l'antenne du CNRS intitulée "Ressources des terroirs - cultures, usages, sociétés ". Sous son autorité, le tir est corrigé et la Coucou figure bien dans l'inventaire breton publié en 1994 . L'effet est immédiat, la volaille accède à la reconnaissance des scientifiques et des organismes agricoles. Cette « officialisation » déclenche l'intérêt d'opérateurs peu coopérants jusque-là... La Chambre d'Agriculture d'Ille-et-Vilaine se rapproche alors de nous pour connaitre nos intentions quant à la valorisation de la race. Leur intention est d'améliorer la race ancienne pour qu'elle corresponde aux critères économiques de production, en diminuant le temps de croissance, et réponde aux nouveaux enjeux de l'aviculture professionnelle. Le refus des éleveurs de Coucou est catégorique : la race doit garder ses qualités originelles de volaille fermière traditionnelle sans autre croisement ou sélection génétique.

En parallèle, nous avons été rejoints par deux ou trois éleveurs professionnels de volailles qui fournissent la grande restauration du département. Ces éleveurs, très engagés dans la qualité, partagent les convictions des éleveurs 
amateurs et engagent des séances de dégustation et des tests qualitatifs avec leurs grands restaurateurs. L'intention est bien d'essayer d'intégrer la filière gastronomique et la grande restauration. Ce noyau dur est rejoint par une bonne dizaine d'éleveurs professionnels soucieux de diversifier leur production. En général, ils appartiennent à la confédération paysanne et défendent une agriculture familiale. Parmi les grands restaurateurs étoilés, figurent Olivier Roellinger à Cancale et Marc Tison à Rennes. Ces chefs, très sensibilisés au patrimoine, vont entrer dans la démarche de manière informelle. Après avoir goûté la volaille, l'enthousiasme est réel. La volonté de développer la Coucou et de la promouvoir va d'abord s'orienter sur les élus de Rennes et les critiques des grands guides gastronomiques. Une dégustation « à l'aveugle " est conduite avec des critiques influents dans un grand restaurant rennais. Le succès est bien là : chacun sort convaincu que la Coucou est doté de qualités gustatives remarquables qu'il convient de remettre sur les tables et les marchés locaux.

Ces éleveurs professionnels et semi-professionnels s'avèrent déterminants dans les discussions avec la Chambre d'agriculture : en préférant garder leur volaille rustique, ils orientent leur projet vers la qualité et le goût. Il faut dire que la Coucou s'élève en 150 jours - quatre à cinq mois - là où les poulets "label » poussent en 50/60 jours... Avec un prix de revient proportionnel à la durée d'élevage... Les producteurs choisissent de rentrer dans une démarche AOC en élevant les volailles sur un parcours herbeux avec une faible densité d'animaux (10 mètres carrés par volaille), décision que la Chambre d'agriculture n'approuve pas, craignant un prix de vente dissuasif. Après avoir longuement hésité, la chambre d'agriculture finit par instruire un dossier administratif et financier qui défend le schéma de production et permet à la micro-filière naissante de bénéficier d'aides substantielles pour sa structuration.

La ville de Rennes fut également un acteur majeur dans la construction de cette filière. L'Ecomusée étant alors un équipement culturel municipal, les élus ont tout de suite adhéré au projet et reconnu la Coucou comme un animal identitaire pour la métropole régionale. Ce capital de sympathie va se traduire par de nombreux articles de presse : journaux municipaux, médias régionaux et nationaux... Autre facteur déterminant, le président des éleveurs professionnels est un «leader paysan » qui pratique la vente directe sur le marché des Lices et s'avère aussi investi dans la vie commerçante de la cité. Paysan militant, il bénéficie de la confiance et du soutien d'un maire soucieux de solidarité entre les territoires, entre la ville et la campagne. L'attente et les encouragements de ce dernier seront réguliers durant près de 15 ans : "Il faut vous lancer et valoriser ce produit... faites attention à la récupération commerciale par l'agroalimentaire et la grande distribution... soyez vigilants et restez exigeants... comptez sur nous pour relayer la communication et soutenir la filière... continuez d'avancer dans le respect de ce patrimoine... vous disposez pour cet accompagnement d'un Ecomusée engagé... ". Ce soutien discret mais efficace d'une municipalité inscrite dans la durée s'est avéré précieux dans l'aventure. 
L'association de producteurs s'est donc créée et engagée dans une démarche de production de qualité à la toute fin des années 90, avec un cahier des charges très précis en termes de surface et aussi en termes d'alimentation pour faire une volaille de qualité. La micro-filière représente aujourd'hui une dizaine d'éleveurs et une production de 25000 volailles, ce qui est peu par rapport aux poulets de Janzé ou de Loué. La démarche de protection et de valorisation est très révélatrice du renouveau des races locales et d'une conservation génétique réussie. Preuve, s'il en est besoin, que des alternatives aux modèles agricoles et économiques dominants existent bien.

Les leçons de l'expérience : une synergie forte entre des acteurs engagés dans le patrimoine, une déontologie très forte des acteurs et des éleveurs professionnels qui veulent rester proches de l'écomusée (lieu de mémoire) et l'intuition majeure de dissocier la conservation de la race et sa production. Autre conclusion, la nécessité de prendre le temps d'écouter, de construire, de structurer la conservation patrimoniale avant d'envisager une valorisation économique. Il y a eu ici un respect du temps, de la mémoire collective et de traditions vivaces qui entretiennent un lien très fort entre la ville et la campagne. Cette dimension de la mémoire active d'une grande ville « campagnarde " se mesure toujours très fortement. Denis Chevallier, ethnologue, avait d'ailleurs souligné ce rôle des races locales (dont la Coucou) dans la réapparition d'identités et de dynamiques locales enracinées dans l'histoire du territoire, y compris urbain à Rennes. L'implication des professionnels de la gastronomie fut une aubaine car ils furent de vrais ambassadeurs, discrets, mais avec de gros carnets d'adresses et beaucoup de relationnel.

Dans cette histoire, l'Écomusée occupe une place centrale car il garantit l'histoire et l'origine du patrimoine. Il y a aussi, il faut bien le reconnaitre, l'engagement et la personnalité des acteurs qui, par leur charisme, leurs convictions et leur implication, furent les éléments-moteurs du projet. Autre élément déterminant de la réussite d'une action, celui de la pérennité des acteurs : après 20 ans le président " historique " quitte ses fonctions cette année et nous espérons que la succession connaitra un second souffle aussi efficace dans l'engagement.

Fin 2013, il est apparu indispensable de restituer cette longue histoire de la Coucou depuis ses origines, et de le faire dans une publication éditée aux Presses Universitaires de Rennes. Ce livre s'imposait, non pour clore un chapitre, mais pour raconter cette mémoire avec la voix et les témoignages des acteurs " historiques ". Volonté peut-être aussi de couper court à une histoire réinventée et aux tentations de récupérations qu'un produit de qualité peut faire naître dans une période en recherche d'images de " terroir " et de produits associés.

Localement la Coucou est devenue l'un des symboles de l'identité du territoire : on la trouve en couverture de journaux rennais ou métropolitains, de quotidiens régionaux, sur des affiches qui l'utilisent comme emblème. 
Idem pour la communication autour des Transmusicales, le grand festival rock rennais, où la coucou de Rennes apparaît sur le programme. Cette récupération institutionnelle et associative est bien sûr positive, mais elle est aussi accompagnée de démarches individuelles et artistiques plus surprenantes. Ainsi, récemment, le grapheur anonyme qui signe ses créations par "WAR » s'est emparé d'une façade pour y peindre trois énormes portraits de Coucou de Rennes. Ne sont-ce pas là des signes tout à fait tangibles d'une réappropriation d'un patrimoine par une génération qui n'a pas connu les cours de fermes traditionnelles d'Ille-et-Vilaine et leurs poulaillers... 